\title{
Alterações hepáticas em trabalhadores de uma refinaria de petróleo e em uma população de referência no Estado da Bahia, Brasil
}

\author{
João Luiz Barberino, ${ }^{1}$ Fernando Martins Carvalho, ${ }^{2}$ Annibal Muniz Silvany-Neto, ${ }^{2}$ \\ Helma Pinchemel Cotrim, ${ }^{2}$ Roberto Charles Góes, ${ }^{3}$ Helton Rosa, ${ }^{3}$ \\ José Florentino Gidi, ${ }^{3}$ Carlos Melgaço Valladares ${ }^{3}$ e Fernando Guedes ${ }^{3}$
}

Forma de citar

Barberino JL, Carvalho FM, Silvany-Neto AM, Cotrim HP, Góes RC, Rosa H, Gidi JF, Valladares CM, Guedes F. Alterações hepáticas em trabalhadores de uma refinaria de petróleo e em uma população de referência no Estado da Bahia, Brasil. Rev Panam Salud Publica. 2005;17(1):30-7.

RESUMO Objetivo. Determinar a prevalência de alterações nas enzimas hepáticas de trabalhadores de uma refinaria de petróleo localizada no Estado da Bahia em comparação a uma população de referência não ocupacionalmente exposta a produtos químicos, e descrever os fatores associados à variação nessa prevalência.

Método. Foram avaliados os trabalhadores da refinaria e os funcionários do escritório da gerência administrativa da empresa, situada em Salvador, capital do Estado da Bahia. Estudaram-se amostras de sangue de 692 trabalhadores da refinaria e de 377 trabalhadores da população de referência. Classificaram-se como casos de alterações hepáticas indivíduos que apresentaram valores acima dos padrões de referência simultaneamente para gama-glutamiltransferase (GGT) (> $50 \mathrm{U} / \mathrm{L}$ para o sexo masculino e $>32 \mathrm{U} / \mathrm{L}$ para o sexo feminino) e alanina aminotransferase $(A L T)(>50 \mathrm{U} / \mathrm{L})$. Foram coletadas informações sobre idade, sexo, peso, altura, tempo de serviço, uso de álcool, hábito de fumar, exercício físico, exposição ocupacional a produtos químicos, uso de equipamento de proteção individual e antecedentes médicos de hepatite, icterícia e obesidade.

Resultados. A prevalência de alterações hepáticas na refinaria foi de 15,3\% (IC95\%: 12,5 a 18,1), contra 3,8\% (IC95\%: 1,8 a 5,8) na população de referência. A análise de regressão logística múltipla estimou que os trabalhadores da refinaria apresentavam uma prevalência de alterações hepáticas 3,56 vezes maior (IC95\%: 1,99 a 6,38) do que a prevalência observada na população de referência, independentemente de outras covariáveis relevantes, como obesidade, prática de exercícios físicos, fumo e bebida alcoólica.

Conclusões. Os resultados sugerem que a exposição ocupacional desempenha um papel importante na determinação das alterações hepáticas nos trabalhadores dessa refinaria de petróleo.

Palavras-chave Alanina aminotransferase, exposição ocupacional, gama-glutamiltransferase.

1 Universidade Estadual de Feira de Santana (UEFS), Feira de Santana (BA), Brasil. Enviar correspondência para este autor no seguinte endereço: Rua Segunda Travessa Nossa Senhora da Vitória 7, Itapoan, CEP 41620-680, Salvador, BA, Brasil. Fone/fax: +55-71-375-3546; e-mail: barberino@ uefs.br
O monitoramento das enzimas hepáticas gama-glutamiltransferase (GGT),

2 Universidade Federal da Bahia (UFBa), Faculdade de Medicina, Salvador (BA), Brasil.

3 Petróleo Brasileiro S.A. - PETROBRÁS, São Francisco do Conde (BA), Brasil. alanina aminotransferase (ALT) e aspartato aminotransferase (AST) é recomendado para a investigação de hepatotoxicidade em trabalhadores industriais (1). Estudos realizados em populações ocupacionalmente expos- 
tas a derivados de petróleo revelaram a existência $(2,3)$ ou a inexistência $(4,5)$ de associação entre as alterações nessas enzimas e a exposição a compostos específicos. Outros estudos identificaram a obesidade (6-8) e o consumo de álcool $(6,9)$ como os principais fatores etiológicos, independentemente da exposição de origem ocupacional. Ho et al. (2) estudaram 271 trabalhadores expostos ocupacionalmente a monômero de cloreto de vinila (MCV) e constataram anormalidades nos testes de função hepática de $13(4,8 \%)$ dos indivíduos estudados. Destes, 12 (92,3\%) apresentaram disfunção hepática induzida pelo MCV. Observaram ainda que, na maioria dos casos, a elevação da ALT sérica foi a primeira alteração enzimática, seguida do aumento da GGT. Essa observação foi fundamental, uma vez que estabeleceu um parâmetro para detectar precocemente as alterações hepáticas, antes do estabelecimento de lesões irreversíveis. Foi observada a redução dos níveis enzimáticos quando os indivíduos afetados foram afastados da exposição.

O trabalho pioneiro de Oliveira et al. (10) em uma refinaria de petróleo no Estado da Bahia, Brasil - a mesma refinaria investigada no presente estudorevelou elevada prevalência de alterações nos níveis séricos de GGT nos exames periódicos de trabalhadores, tendo sido essas alterações associadas ao consumo de álcool. Uma análise subseqüente (11) desse banco de dados estimou que a prevalência de GGT elevada no universo dos 1912 trabalhadores da refinaria era de $38,0 \%$. A prevalência de GGT elevada foi maior nos grupos com maior consumo de álcool e com exposição ocupacional a gases, a solventes ou a produtos químicos. Entre os não-bebedores, $16 \%$ tinham GGT elevada. Informações coletadas nos prontuários indicaram que, neste grupo, a prevalência de GGT elevada estava associada à exposição ocupacional a gases [razão de prevalência (RP) em relação aos não expostos $=10,9]$, a produtos químicos $(\mathrm{RP}=3,5)$ e a solventes $(R P=1,9)$. Apesar de instigantes, essas associações devem ser consideradas de forma cautelosa, devido às limitações no desenho do estudo que as gerou e ao efeito de vieses diversos.
Em 1995, foram diagnosticados 10 casos de esteato-hepatite não-alcoólica sugestiva de agressão tóxica em trabalhadores industriais do Estado da Bahia, alguns deles procedentes da refinaria em estudo (12). A exposição a produtos químicos foi considerada como um possível fator etiológico. Em 1996 (13), a casuística se elevou para 15 casos e, em 1997 (14), para 20 casos; a exposição a produtos químicos industriais e a substâncias petroquímicas voláteis foi mencionada como possível fator etiológico. Em outro estudo (15), foram realizadas avaliações mensais seriadas de GGT, ALT e AST e duas biópsias hepáticas, com intervalo de 12 meses, em nove casos com esteato-hepatite nãoalcoólica que concordaram em se afastar do trabalho. O acompanhamento permitiu observar um decréscimo gradual dessas enzimas nos nove indivíduos após o afastamento do ambiente de trabalho. Histologicamente, as estruturas hepáticas também melhoraram, em todos os casos.

A análise de dados obtidos no arquivo do serviço de saúde ocupacional da refinaria em estudo permitiu, ainda, a descrição (16) de uma série de 548 casos, definidos pela alteração acima de $10 \%$ do valor de referência nas dosagens de GGT, ALT, AST, bilirrubina direta (BD), bilirrubina indireta $(\mathrm{BI})$, bilirrubina total $(\mathrm{BT})$ ou fosfatase alcalina (FA). Verificou-se que a alteração isolada de GGT e a alteração simultânea de GGT e ALT foram as mais freqüentes, observadas em 72,4 e $41,4 \%$ do grupo, respectivamente. A elevação simultânea de GGT, ALT e AST foi observada em 29,7\% dos casos. Chamou a atenção o fato de que 92,5\% dos indivíduos no grupo estudado trabalhavam em setores da produção e $36,5 \%$ tinham mais de 20 anos de trabalho na refinaria. Foram anotados os antecedentes médicos de hepatite $(8,6 \%)$, icterícia $(7,1 \%)$, esquistossomose $(23,2 \%)$, transfusão sanguínea $(1,4 \%)$, obesidade $(30,1 \%)$ e uso diário ou freqüente de álcool $(17,8 \%)$. Nenhuma dessas características foi referida por $26,0 \%$ dos trabalhadores, o que sugere a possibilidade de etiologias ocupacionais para as alterações hepáticas apresentadas.
O objetivo do presente estudo foi determinar a prevalência de alterações hepáticas em trabalhadores dessa refinaria de petróleo em comparação a uma população não ocupacionalmente exposta a produtos químicos, e identificar os fatores associados à variação nessa prevalência.

\section{MATERIAIS E MÉTODOS}

Foi realizado um estudo de prevalência que incluiu uma população de comparação externa (17). A refinaria estudada iniciou suas atividades em 1950, antecedendo a criação da Petrobrás. Ocupa uma área de cerca de 3,7 milhões de $\mathrm{m}^{2}$ e em 1997 processava 140000 barris de petróleo por dia. Naquele ano, a refinaria tinha cerca de 1660 trabalhadores com vínculo direto, distribuídos em 44 setores. A refinaria processa uma série de compostos químicos relacionados ao refino do petróleo: hidrocarbonetos, compostos de nitrogênio e enxofre, ácidos, bases, carbonatos, álcoois, cetonas, antimônio, cloro e metais. A exposição dos trabalhadores é bastante variada, indo desde o contato direto até o contato acidental em laboratório.

Como população de referência foi tomada uma amostra de trabalhadores da gerência administrativa da empresa na Bahia (GERAB), localizada na Cidade de Salvador. Considerou-se como caso de alteração hepática o indivíduo que apresentou valores séricos acima dos níveis considerados normais, de acordo com o método de dosagem utilizado, simultaneamente para as enzimas GGT ( $>50 \mathrm{U} / \mathrm{L}$ para o sexo masculino e $>32 \mathrm{U} / \mathrm{L}$ para o sexo feminino) e ALT (> $50 \mathrm{U} / \mathrm{L}$ ).

Com base em estudos anteriores, foi assumida uma prevalência de $15 \%$ de elevação na GGT e ALT na refinaria. Para a GERAB, a prevalência assumida, com base em estudos sobre os valores normais na população geral, foi de 5\%. Uma amostra de no mínimo 188 indivíduos seria necessária em cada grupo para garantir um nível de confiança de $95 \%$ e um poder de $90 \%$ (teste bicaudal) (18). Esses números amostrais foram ampliados para 692 
indivíduos na refinaria (fração amostral igual a $41,61 \%$ ) e para 377 indivíduos na GERAB (fração amostral igual a $53,22 \%$ ), em função da disponibilidade operacional e visando o aumento do poder do estudo.

Um questionário padronizado contendo informações epidemiológicas relevantes foi aplicado a cada trabalhador no momento do exame anual nas duas populações estudadas. A equipe de coleta de dados foi composta de médicos da refinaria e da GERAB. Visando a padronização dos procedimentos, foi realizado o treinamento da equipe através de discussão prévia dos instrumentos, entrevistas simuladas e coleta de dados em prontuários, para posterior discussão. A coleta na refinaria foi de maio de 1997 a fevereiro de 1998, e na GERAB, de março de 1998 a outubro de 1998.

Em relação ao uso de álcool, além das perguntas "bebe", "freqüência com que bebe" e "tipo de bebida que consome", foi também utilizado o teste CAGE (19). Com base na freqüência de uso de bebidas destiladas e fermentadas, foi criada, também, a categoria "grandes bebedores". Foram considerados grandes bebedores os indivíduos que faziam uso diário ou freqüente (2 a 6 dias na semana) de bebidas alcoólicas destiladas ou fermentadas. Indivíduos que fumavam 20 ou mais cigarros por dia foram considerados como grandes fumantes. Indivíduos que consumiam mais de 100 $\mathrm{mL}$ de café por dia foram classificados como grandes bebedores de café.

$\mathrm{O}$ índice de massa corporal (IMC; peso em $\mathrm{kg}$ dividido pelo quadrado da altura em m) foi calculado para cada trabalhador a partir de informações coletadas nos respectivos prontuários.

Coletaram-se amostras de sangue dos grupos exposto e de referência para dosagens séricas de GGT, ALT, AST, BD, BI e colesterol. Foram despendidos esforços no sentido de padronizar os métodos laboratoriais utilizados para avaliação da função hepática dos trabalhadores da refinaria (Laboratório da Fundação José Silveira) e GERAB (Laboratório do Serviço Social da Indústria). Esses laboratórios já realizavam intercalibração mensal para controle de qualidade com a Sociedade Brasileira de Patologia Clínica e com a Sociedade Brasileira de Análises Clínicas. O laboratório da Fundação José Silveira obteve um percentual de acerto igual a $91 \%$ em 1997, e em 1998 elevou sua acurácia para $93 \%$. O laboratório do Serviço Social da Indústria apresentou um percentual de acerto que variou de 79,8 a $89 \%$ de janeiro a dezembro de 1998 , sendo a média anual igual a 83,53\%.

Neste estudo, a exposição ocupacional foi definida como o contato direto ou indireto, físico, químico ou físicoquímico, ambiental, em qualquer intensidade ou duração, com agentes que pudessem levar às alterações hepáticas acima definidas em decorrência do trabalho na refinaria. Assim, a refinaria foi considerada como área exposta e a gerência administrativa como área não exposta ou de referência.

As informações profissiográficas (perfil histórico-laboral do trabalhador) constantes nos bancos de dados da empresa permitiram classificar a população da refinaria segundo os seguintes postos de trabalho: operação, manutenção, segurança industrial, segurança patrimonial e movimentação/ transporte/estocagem.

Como passo inicial da análise, foram comparadas as características dos grupos exposto e de referência. As razões de prevalência (RP) das alterações hepáticas foram calculadas a partir da comparação entre os dois grupos. A inferência estatística foi feita pelo teste do qui-quadrado $\left(\chi^{2}\right)$ e pelo teste $t$, sendo determinado o valor de $P$, ou pelo cálculo dos intervalos de confiança (IC) das razões de prevalência, utilizando-se as séries de Taylor. A seguir, realizou-se uma análise estratificada para avaliar a presença de modificação de efeito (interação) ou confundimento. Nessa etapa, as RP foram ajustadas pelo método de MantelHaenszel (20).

A análise de regressão logística múltipla utilizou os procedimentos recomendados por Hosmer e Lemeshow (21). As estimativas da razão de chances (odds ratio, OR), obtidas na análise multivariada, foram convertidas para $\mathrm{RP}$, com seus respectivos IC de $95 \%$
(IC95\%), utilizando-se o método delta (22) através do programa estatístico SAS versão 6.12 (23). Os demais dados foram processados eletronicamente em microcomputadores, usando-se o pacote estatístico SPSS (24).

O estudo foi aprovado pelo Comitê de Ética em Pesquisa do Hospital Universitário Professor Edgard Santos da Universidade Federal da Bahia (UFBa).

\section{RESULTADOS}

A proporção de trabalhadores do sexo masculino na população da refinaria $(93,9 \%$; 650/692) diferia significativamente $(P<0,05)$ daquela na população de referência $(61,5 \% ; 232$ / 377). A idade dos indivíduos nas populações estudadas variou de 23 a 67 anos, com média de 37,4 \pm 6,1 anos para a população exposta e de 41,0 \pm 5,9 anos para a população de referência, sendo essa diferença também estatisticamente significativa $(P<0,05)$.

A tabela 1 mostra que a proporção de indivíduos obesos ou com sobrepeso foi significativamente mais alta na refinaria do que na GERAB. As populações comparadas não diferiram quanto à prática e à freqüência de exercícios físicos, exceto quando foram comparados os trabalhadores que se exercitavam 1 vez por semana àqueles que não praticavam exercícios físicos.

A tabela 2 mostra que o uso de bebida alcoólica foi estatisticamente mais referido por trabalhadores da refinaria do que da GERAB. A proporção de grandes bebedores, assim como o teste CAGE positivo, foi também estatisticamente maior na refinaria. Não foi possível quantificar o uso de álcool em gramas diários devido a sérias distorções, a exemplo do número excessivo de registros perdidos ou considerados inconsistentes. A proporção de trabalhadores que consumiam café foi estatisticamente semelhante nas duas populações; contudo, o volume médio ingerido diariamente foi significativamente maior nos trabalhadores da refinaria do que na GERAB. A proporção de fumantes na refinaria foi estatisticamente semelhante àquela encontrada 
TABELA 1. Distribuição dos trabalhadores de uma refinaria de petróleo e da população de referência segundo o índice de massa corporal e a prática de exercícios físicos, Estado da Bahia, Brasil, 1997 e 1998

\begin{tabular}{|c|c|c|c|c|c|}
\hline \multirow[b]{2}{*}{ Covariável } & \multicolumn{2}{|c|}{ Refinaria } & \multicolumn{2}{|c|}{$\begin{array}{c}\text { População } \\
\text { de referência }\end{array}$} & \multirow[b]{2}{*}{$P$} \\
\hline & No. & $\%$ & No. & $\%$ & \\
\hline \multicolumn{6}{|c|}{ Índice de massa corporal $\left(\mathrm{kg} / \mathrm{m}^{2}\right)$} \\
\hline Normal (até 25,99) & 331 & 48,5 & 220 & 58,8 & Referência \\
\hline Sobrepeso $(26$ a 29,99$)$ & 274 & 40,2 & 124 & 33,2 & $<0,01$ \\
\hline Obeso $(\geq 30,0)$ & 77 & 11,3 & 30 & 8,0 & $<0,05$ \\
\hline Total & 682 & 100,0 & 374 & 100,0 & \\
\hline \multicolumn{6}{|c|}{ Prática regular de exercícios físicos } \\
\hline Não & 343 & 50,5 & 212 & 56,2 & Referência \\
\hline Sim & 336 & 49,5 & 165 & 43,8 & $>0,05$ \\
\hline Total & 679 & 100,0 & 377 & 100,0 & \\
\hline \multicolumn{6}{|c|}{ Freqüência com que se exercita } \\
\hline Não se exercita & 343 & 50,5 & 212 & 56,2 & Referência \\
\hline Diariamente & 76 & 11,2 & 39 & 10,3 & $>0,05^{b}$ \\
\hline 2 a 4 vezes por semana & 193 & 28,4 & 104 & 27,6 & $>0,05^{b}$ \\
\hline 1 vez por semana & 54 & 8,0 & 16 & 4,3 & $<0,05^{b}$ \\
\hline Raramente & 13 & 1,9 & 6 & 1,6 & $>0,05^{b}$ \\
\hline Total & 679 & 100,0 & 377 & 100,0 & \\
\hline
\end{tabular}

a Trabalhadores da gerência administrativa da empresa.

b Comparando a proporção de indivíduos em cada categoria de freqüência de exercício com a proporção de indivíduos que não praticavam exercícios na refinaria e na gerência administrativa.

TABELA 2. Distribuição dos trabalhadores de uma refinaria de petróleo e da população de referência segundo o uso de álcool e café e o hábito de fumar, Estado da Bahia, Brasil, 1997 e 1998

\begin{tabular}{|c|c|c|c|c|c|}
\hline \multirow[b]{2}{*}{ Covariável } & \multicolumn{2}{|c|}{ Refinaria } & \multicolumn{2}{|c|}{$\begin{array}{c}\text { População } \\
\text { de referência }\end{array}$} & \multirow[b]{2}{*}{$P$} \\
\hline & No. & $\%$ & No. & $\%$ & \\
\hline Bebe & 567 & 82,2 & 250 & 66,3 & $<0,05$ \\
\hline \multicolumn{6}{|l|}{ Tipo de bebida } \\
\hline Fermentada & 518 & 74,9 & 243 & 64,5 & $<0,05$ \\
\hline Destilada & 127 & 18,8 & 28 & 7,4 & $<0,05$ \\
\hline Grandes bebedores $^{b}$ & 72 & 11,9 & 5 & 1,3 & $<0,05$ \\
\hline Teste CAGE positivo & 101 & 14,9 & 11 & 2,9 & $<0,05$ \\
\hline Uso de café & 496 & 72,0 & 284 & 75,3 & $>0,05$ \\
\hline \multicolumn{6}{|l|}{ Ingestão diária de café } \\
\hline (média \pm desvio-padrão em mL) & \multicolumn{2}{|c|}{$261 \pm 185$} & \multicolumn{2}{|c|}{$190 \pm 172$} & $<0,05$ \\
\hline Fumante atual & 111 & 16,2 & 47 & 12,5 & $>0,05$ \\
\hline \multirow{3}{*}{$\begin{array}{l}\text { Número de cigarros por dia } \\
\text { (média } \pm \text { desvio-padrão em } \mathrm{mL} \text { ) } \\
\text { Grandes fumantes }{ }^{c}\end{array}$} & \multirow{2}{*}{\multicolumn{2}{|c|}{$13,6 \pm 9,2$}} & \multirow{2}{*}{\multicolumn{2}{|c|}{$9,5 \pm 6,7$}} & \\
\hline & & & & & $<0,05$ \\
\hline & 40 & 5,8 & 10 & 2,7 & $<0,05$ \\
\hline
\end{tabular}

a Trabalhadores da gerência administrativa da empresa.

${ }^{\mathrm{b}}$ Consumo de álcool diário ou de 2 a 6 dias por semana.

c 20 ou mais cigarros por dia.

na GERAB. Contudo, a intensidade desse hábito foi estatisticamente maior entre os trabalhadores da refinaria, considerando o maior número médio de cigarros fumados por dia e a maior proporção de grandes fumantes.
A proporção de trabalhadores com antecedente médico de hepatite não diferiu $(P>0,05)$ entre trabalhadores da refinaria $(8,7 \%)$ e da GERAB $(10,3 \%)$. Já o antecedente de "outras doenças" foi significativamente $(\mathrm{P}<$
$0,05)$ mais freqüente nos trabalhadores da refinaria $(36,6 \%)$ do que nos da $\operatorname{GERAB}(28,5 \%)$.

A prevalência de exames acima dos limites de referência foi significativamente mais elevada na refinaria para todos os marcadores, com exceção de BT (tabela 3). A prevalência de alterações hepáticas (GGT e ALT elevados) também foi significativamente maior entre trabalhadores da refinaria, com RP igual a 4,02 (IC95\%: 2,29 a 7,07).

A tabela 4 revela que a prevalência de alterações hepáticas entre trabalhadores do setor administrativo da refinaria foi estatisticamente semelhante à da população de referência. Os trabalhadores do setor produtivo da refinaria estavam mais afetados.

Na refinaria, a prevalência de alterações hepáticas foi de 18,2\% entre os empregados com tempo de serviço acima do valor mediano (13,24 anos) e de $12,5 \%$ entre os que trabalhavam há 13,24 anos ou menos, tendo essa diferença apresentado significância limítrofe $(P=0,059)$. Na GERAB, a prevalência foi semelhante para os dois tempos de exposição: $3,7 \%$ para o grupo de empregados com maior tempo de serviço e 3,9\% entre os que se encontravam na mediana do tempo de serviço (13,73 anos) ou abaixo dela $(P>0,05)$. Na análise estratificada, verificou-se que, dentre todas as covariáveis investigadas, apenas o antecedente médico de hepatite e o antecedente médico de outras doenças modificavam o efeito da associação principal. Nenhuma das interações, contudo, foi confirmada na análise multivariada.

Os resultados da avaliação tabular para potenciais confundidores evidenciaram que apenas as variáveis uso de álcool (bebe) e uso de bebidas fermentadas apresentaram-se como possíveis confundidoras.

A Tabela 5 revela que a associação entre o trabalho na refinaria e as alterações hepáticas foi mais forte entre os não-bebedores do que entre os bebedores. Essa associação também foi mais forte entre os que não usavam bebidas fermentadas do que entre os que as usavam. Após o ajuste por análise es- 
TABELA 3. Prevalência de exames laboratoriais com valores elevados em trabalhadores de uma refinaria de petróleo e na população de referência, Estado da Bahia, Brasil, 1997 e 1998

\begin{tabular}{|c|c|c|c|c|c|c|c|}
\hline \multirow[b]{2}{*}{ Exame $^{b}$} & \multicolumn{3}{|c|}{ Refinaria } & \multicolumn{3}{|c|}{ População de referência ${ }^{a}$} & \multirow[b]{2}{*}{$P$} \\
\hline & Examinados & Casos & $\%$ & Examinados & Casos & $\%$ & \\
\hline GGT & 650 & 199 & 30,6 & 351 & 73 & 20,8 & $<0,001$ \\
\hline ALT & 651 & 136 & 20,9 & 361 & 22 & 6,1 & $<0,00001$ \\
\hline AST & 651 & 82 & 12,6 & 346 & 9 & 2,5 & $<0,00001$ \\
\hline $\mathrm{BD}$ & 642 & 77 & 12,0 & 346 & 9 & 2,6 & $<0,0001$ \\
\hline $\mathrm{BI}$ & 641 & 50 & 7,8 & 347 & 51 & 14,7 & $<0,001$ \\
\hline BT & 640 & 48 & 7,5 & 347 & 34 & 9,8 & $>0,05$ \\
\hline Colesterol & 640 & 317 & 49,5 & 362 & 54 & 14,9 & $<0,00001$ \\
\hline GGT + ALT (alterações hepáticas) & 649 & 98 & 15,1 & 342 & 13 & 3,8 & $<0,00001$ \\
\hline
\end{tabular}

a Trabalhadores da gerência administrativa empresa.

b Valores de referência: GGT (gama-glutamiltransferase): $>50 \mathrm{U} / \mathrm{L}$ para o sexo masculino e > $32 \mathrm{U} / \mathrm{L}$ para o sexo feminino; ALT (alanina aminotransferase): > $50 \mathrm{U} / \mathrm{L}$; AST (aspartato aminotransferase): > $46 \mathrm{U} / \mathrm{L} ; \mathrm{BD}$ (bilirrubina direta): > 4,27 $\mu \mathrm{mol} / \mathrm{L} ; \mathrm{Bl}$ (bilirrubina indireta): > 12,82 $\mu \mathrm{mol} / \mathrm{L} ; \mathrm{BT}$ (bilirrubina total): > 17,0 $\mu \mathrm{mol} / \mathrm{L} ; \mathrm{colesterol}:>6,22 \mathrm{mmol} / \mathrm{L}$.

tratificada, ambas as RP se reduziram substancialmente $(\mathrm{RP}=1,39$ e 1,36 , respectivamente), mas os IC95\% continuaram maiores do que a unidade.

Procedeu-se, então à análise de regressão logística múltipla em um modelo incluindo inicialmente as variáveis local de trabalho, idade, sexo, consumo de bebidas alcoólicas, uso de álcool no passado, consumo de bebidas fermentadas, consumo de bebidas destiladas, teste CAGE, grande consumo de álcool, hábito de fumar, condição de ex-fumante, uso diário de café, grande consumo de café, exercício físico regular, freqüência do exercício, obesidade, antecedente médico de obesidade, antecedente médico de icterícia, antecedente médico de hepatite, antecedente médico de transfusão sangüínea, ante- cedente médico de outras doenças, uso regular de medicamentos, tempo de serviço, uso de equipamentos de proteção individual, referência a exposição atual a produtos químicos e exposição a produtos químicos no passado. Foram também considerados na análise os termos de interação de primeira ordem envolvendo a variável independente principal (alterações hepáticas) e cada uma das covariáveis, desde que os mesmos tivessem plausibilidade biológica. Foram mantidas no modelo final as variáveis local de trabalho, prática de exercícios físicos, hábito de fumar, obesidade e consumo de bebidas alcoólicas.

O modelo estimou que os trabalhadores da refinaria apresentavam prevalência 3,56 vezes maior de alterações hepáticas (IC95\%: 1,99 a 6,38) do que a população de referência, após controle dos efeitos das demais covariáveis presentes no modelo (tabela 6). A obesidade, o uso de álcool e a prática de exercícios físicos se associaram fortemente à prevalência de alterações hepáticas. O hábito de fumar apresentou significância estatística limítrofe $(P=$ $0,0610)$. O diagnóstico da regressão e a análise de resíduos mostraram adequação do modelo final (teste da razão de verossimilhança: $P=1,0000$; qualidade do ajuste: $P=0,9560$ ). Não foi necessária a retirada de casos anômalos ou muito influentes.

A avaliação para confundimento através da análise de regressão logística múltipla não evidenciou, em nenhum dos modelos testados, confundi-

TABELA 4. Prevalência de alterações hepáticas em trabalhadores de uma refinaria de petróleo e na população de referência segundo o setor de trabalho, Estado da Bahia, Brasil, 1997 e 1998

\begin{tabular}{|c|c|c|c|c|c|c|c|}
\hline \multirow[b]{2}{*}{ Setor de trabalho } & \multicolumn{3}{|c|}{ Refinaria } & \multicolumn{3}{|c|}{ População de referência ${ }^{a}$} & \multirow[b]{2}{*}{$P$} \\
\hline & No. & Casos & $\%$ & No. & Casos & $\%$ & \\
\hline Administrativo & 84 & 3 & 3,6 & 346 & 13 & 3,8 & $>0,05$ \\
\hline Produção & 564 & 95 & 16,8 & - & - & - & - \\
\hline Manutenção & 104 & 17 & 16,3 & - & - & - & 一 \\
\hline Operação & 385 & 70 & 18,2 & - & - & - & 一 \\
\hline Movimentação/Transporte/Estocagem & 12 & 4 & 33,3 & - & - & - & - \\
\hline Segurança industrial & 5 & 0 & 0,0 & - & - & - & - \\
\hline Segurança patrimonial & 4 & 0 & 0,0 & - & - & - & - \\
\hline Laboratório & 54 & 4 & 7,4 & - & - & - & - \\
\hline Total & 648 & 98 & 15,1 & 346 & 13 & 3,8 & $<0,05$ \\
\hline
\end{tabular}

a Trabalhadores da gerência administrativa da empresa. 
TABELA 5. Associação entre o trabalho em uma refinaria de petróleo e as alterações hepáticas ajustada para uso de bebida alcoólica e uso de bebida alcoólica fermentada, Estado da Bahia, Brasil, 1997 e 1998

\begin{tabular}{lccc}
\hline \multicolumn{1}{c}{ Covariável } & No. & Razão de prevalências & IC95\% \\
\hline Associação bruta & 994 & 4,02 & 2,29 a 7,07 \\
Bebe & 763 & 3,06 & 1,75 a 5,37 \\
$\quad$ Sim & 229 & 8,62 & 1,11 a 66,97 \\
Não & 992 & 1,39 & 1,20 a 1,61 \\
$\quad$ Após ajuste & & 3,39 & 1,89 a 6,06 \\
Bebida fermentada & 712 & 8,55 & 1,10 a 66,38 \\
Sim & 228 & 1,36 & 1,17 a 1,57 \\
Não & 940 & & \\
Após ajuste & & & \\
\hline
\end{tabular}

mento da associação principal pelas covariáveis do modelo final da análise de regressão (prática de exercícios físicos, hábito de fumar, obesidade e uso de álcool) (tabela 7).

\section{DISCUSSÃO}

Foi encontrada uma forte associação entre alterações simultâneas nas enzimas hepáticas GGT e ALT e trabalho na refinaria, estando controlados os efeitos de outras covariáveis usualmente investigadas na prática médica

TABELA 6. Resultados da análise de regressão logística múltipla sobre a associação entre as alterações hepáticas e a exposição ocupacional em trabalhadores de refinaria de petróleo, Estado da Bahia, Brasil, 1997 e 1998

\begin{tabular}{|c|c|c|c|c|c|c|}
\hline Covariável $^{a}$ & $\beta$ & Erro-padrão & $P$ & OR & $\begin{array}{c}\text { Razão de } \\
\text { prevalências }\end{array}$ & IC95\% \\
\hline Local de trabalho & 1,3443 & 0,3099 & $<0,001^{b}$ & 3,83 & 3,56 & 1,99 a 6,38 \\
\hline Exercício & $-0,5476$ & 0,2295 & $=0,0150^{\mathrm{b}}$ & 0,60 & 0,59 & 0,39 a 0,91 \\
\hline Fuma & 0,5085 & 0,2644 & $=0,0610^{b}$ & 1,66 & 1,63 & 0,99 a 2,67 \\
\hline Obesidade & 1,2301 & 0,2624 & $<0,0001^{\mathrm{b}}$ & 3,42 & 3,24 & 2,00 a 5,24 \\
\hline Bebe & 1,1024 & 0,3860 & $<0,0013^{b}$ & 3,01 & 2,85 & 1,34 a 5,99 \\
\hline Constante & $-4,1307$ & 0,4417 & $<0,0001^{\mathrm{c}}$ & - & - & \\
\hline
\end{tabular}

Local: $0=$ gerência administrativa, $1=$ refinaria. Exercício: $0=$ não pratica, $1=$ pratica. Fuma: $0=$ não, $1=$ sim. Obesidade $($ IMC $\geq 30): 0=$ não, $1=$ sim. Bebe: $0=$ não, $1=$ sim.

b Teste da razão de verossimilhança.

c Estatística de Wald.

TABELA 7. Análise multivariada da magnitude e direção do confundimento da associação entre o trabalho em uma refinaria de petróleo e as alterações hepáticas segundo diversas covariáveis, Estado da Bahia, Brasil, 1997 e 1998

\begin{tabular}{|c|c|c|c|c|c|c|}
\hline Modelos $^{a}$ & $B_{1}$ & OR & $P$ & Razão de prevalências & $\mathrm{IC} 95 \%$ & $\Delta \mathrm{RP}_{\mathrm{AJ}-\mathrm{NAJ}} \mathrm{b}^{\mathrm{b}}$ \\
\hline Local de trabalho & 1,5182 & 4,5641 & $<0,0001$ & 4,016 & 2,284 a 7,076 & Não-ajustada \\
\hline Local + exercício & 1,5265 & 4,6018 & $<0,0001$ & 3,988 & 2,262 a 7,031 & 0,028 \\
\hline Local + fuma & 1,5114 & 4,5331 & $<0,0001$ & 3,961 & 2,251 a 6,970 & 0,055 \\
\hline Local + obesidade & 1,4741 & 4,3673 & $<0,0001$ & 3,929 & 2,237 a 6,902 & 0,087 \\
\hline Local + bebe & 1,4055 & 4,0777 & $<0,0001$ & 3,832 & 2,138 a 6,869 & 0.184 \\
\hline Local + exercício + fuma & 1,5148 & 4,5486 & $<0,0001$ & 3,879 & 2,214 a 6,797 & 0,137 \\
\hline Local + exercício + obesidade & 1,4841 & 4,4110 & $<0,0001$ & 3,762 & 2,150 a 6,580 & 0,254 \\
\hline Local + exercício + bebe & 1,3826 & 3,9851 & $<0,0001$ & 3,706 & 2,069 a 6,637 & 0,310 \\
\hline Local + fuma + obesidade & 1,4577 & 4,2959 & $<0,0001$ & 3,730 & 2,123 a 6,553 & 0,286 \\
\hline Local + fuma + bebe & 1,4052 & 4,0762 & $<0,0001$ & 3,812 & 2,126 a 6,834 & 0,204 \\
\hline Local + obesidade + bebe & 1,3635 & 3,9100 & $<0,0001$ & 3,692 & 2,058 a 6,625 & 0,324 \\
\hline $\begin{array}{l}\text { Local + exercício + fuma + } \\
\text { + obesidade }\end{array}$ & 1,4639 & 4,3227 & $<0,0001$ & 3,676 & 2,101 a 6,434 & 0,340 \\
\hline $\begin{array}{l}\text { Local + exercício + fuma + } \\
\quad+\text { bebe }\end{array}$ & 1,3826 & 3,9853 & $<0,0001$ & 3,689 & 2,060 a 6,606 & 0,327 \\
\hline $\begin{array}{l}\text { Local + exercício + obesidade + } \\
\quad+\text { bebe } \\
\text { Local + fuma + obesidade + }\end{array}$ & 1,3505 & 3,8592 & $<0,0001$ & 3,610 & 2,014 a 6,472 & 0,406 \\
\hline $\begin{array}{l}\text { + bebe } \\
\text { Local + fuma + exercício + }\end{array}$ & 1,3566 & 3,8828 & $<0,0001$ & 3,637 & 2,028 a 6,522 & 0,379 \\
\hline + obesidade + bebe & 1,3443 & 3,8356 & $<0,0001$ & 3,562 & 1,988 a 6,382 & 0,454 \\
\hline
\end{tabular}

${ }^{a}$ Local: $0=$ gerência administrativa, $1=$ refinaria. Exercício: $0=$ não pratica, $1=$ pratica. Fuma: $0=$ não, $1=$ sim. Obesidade (IMC $\left.\geq 30\right): 0=$ não, $1=$ sim. Bebe: $0=$ não, $1=$ sim.

${ }^{\mathrm{b}} \Delta \mathrm{RP}_{\mathrm{AJ}-\mathrm{NAJ}}=$ diferença entre razão de prevalências ajustada e não-ajustada. 
ocupacional, tais como obesidade, sedentarismo, fumo e álcool.

No presente estudo, observou-se que, além do trabalho na refinaria, a obesidade apresentou forte associação com as alterações hepáticas. Burns et al. (6), estudando alterações nas enzimas hepáticas GGT, ALT e AST de cerca de 1600 trabalhadores da Dow Chemical, nos Estados Unidos, identificaram a obesidade como o principal fator de risco, embora não tenham investigado a exposição ocupacional. Outros estudos (7-9) enfatizaram o papel da obesidade, álcool, fumo e exercícios físicos na ocorrência de alterações nas enzimas hepáticas, sem avaliar o papel da exposição ocupacional.

Os resultados do presente estudo sugerem que, além dos fatores de risco eminentemente biológicos ou comportamentais, a exposição ocupacional pode desempenhar um papel importante na determinação das alterações hepáticas em trabalhadores do refino de petróleo. A maior prevalência de alterações hepáticas nos indivíduos que trabalham há mais tempo na área exposta sugeriu o efeito de exposição cumulativa. Esses achados, contudo, não se confirmaram na análise de regressão logística. Tais resultados sugerem a necessidade da realização de novos estudos, preferencialmente longitudinais, que possam estabelecer mais claramente a existência ou não de associação entre essas variáveis.

A comparação da refinaria com a gerência administrativa teve o intuito de evitar ou reduzir ao máximo o efeito do trabalhador sadio, freqüentemente observado em trabalhos (17) que estudam a morbi-mortalidade de trabalhadores da indústria em comparação com a população geral. Em nosso estudo, as populações observadas, embora trabalhem em locais diferentes, atuam numa mesma empresa e foram selecionadas pelos mesmos critérios, tendo, portanto, como principal diferença o local de trabalho e, possivelmente, a exposição.

A discussão apresentada nos permite concluir que existe uma maior prevalência de alterações hepáticas entre os trabalhadores da refinaria quando comparados aos trabalhadores da GERAB. Os resultados sugerem que essa diferença se deve à exposição ocupacional a produtos químicos. É importante salientar que a exposição ocupacional típica em uma refinaria de petróleo é muito difícil de ser caracterizada pormenorizadamente, pela grande diversidade de substâncias hepatotóxicas presentes nesse ambiente de trabalho. O setor de higiene industrial da refinaria vinha monitorando regularmente as concentrações atmosféricas de compostos, sem encontrar maiores inconformidades com os limites previstos na legislação ocupacional prevalente no Brasil. ${ }^{4,5}$ Não obstante, tais limites não contemplam o problema da múltipla exposição nem a questão da suscetibilidade individual, que podem determinar agressões tóxicas mesmo que os produtos químicos, dispersos no ambiente do trabalho, estejam dentro dos limites de tolerância biológica. Assim, é necessário caracterizar especificamente a natureza da exposição ocupacional neste e em outros ambientes de trabalho para facilitar o seu controle e minimizar os riscos e danos à saúde dos trabalhadores.

Agradecimentos. $\mathrm{O}$ estudo recebeu apoio financeiro do Conselho Nacional de Desenvolvimento Científico e Tecnológico $(\mathrm{CNPq}) /$ Plano Nacional de Ciência e Tecnologia de Petróleo e Gás Natural (CTPETRO) e da Petrobrás.

\footnotetext{
4 PETROBRÁS. Relatório de monitorização rotineira de poluentes atmosféricos na refinaria, conforme legislação brasileira de higiene e segurança do trabalho [documento mimeografado]. Salvador: 1996.

5 PETROBRÁS. Monitorização de agentes químicos na RLAM [documento mimeografado]. Salvador: 1999.
}

\section{REFERÊNCIAS}

1. Tamburro CH, Liss GM. Tests for hepatotoxicity: usefulness in screening workers. J Occup Med. 1986;28(10):1034-44.

2. Ho SF, Phoon WH, Gan SL, Chan YK. Persistent liver dysfunction among workers at a vinyl chloride monomer polymerization plant. J Soc Occup Med. 1991;41(1):10-6.

3. Guzelian P, Mills S, Fallon HJ. Liver structure and function in print workers exposed to toluene. J Occup Med. 1988;30(10):791-6.

4. Boogaard PJ, Rocchi PSJ, Sittert NJ. Effects of exposure to low concentrations of chlorinated hydrocarbons on the kidney and liver of industrial workers. Br J Ind Med. 1993;50(4): 331-9.

5. Gennari P, Naldi M, Motta R, Nucci MC, Giacomini C, Violante FS, et al. Gammaglutamyltransferase isoenzyme pattern in workers exposed to tetrachloroethylene. Am J Ind Med. 1992;21(5):661-71.

6. Burns CJ, Boswell JM, Olsen GW. Liver enzyme activity and body mass index. J Occup Environ Med. 1996;38(12):1248-52.
7. Hodgson MJ, Thiel DH, Lauschus K, Karpf M. Liver injury tests in hazardous waste workers: the role of obesity. J Occup Med. 1989;31(3): 239-42.

8. Robinson D, Whitehead TP. Effect of body mass and other factors on serum liver enzyme levels in men attending for well population screening. Ann Clin Biochem. 1989;26(Pt 5): 393-400.

9. Rees D, Soderlund N, Cronje R, Song E, Kielkowski D, Myers J. Solvent exposure, alcohol consumption and liver injury in workers manufacturing paint. Scand J Work Environ Health. 1993;19(4):236-44.

10. Oliveira JFG, Torres PB, Menezes JM, Santos EAJ, Góes RC. Utilização da dosagem da gama-glutamil-transferase associada ao teste CAGE no diagnóstico do alcoolismo na empresa. Gramado: Congresso da Associação Brasileira de Estudos do Álcool e Outras Drogas; 1987.

11. Lima CTS. Alcoolismo e doenças associadas. Um estudo de coorte em trabalhadores de uma refinaria de petróleo [dissertação]. Salvador: Universidade Federal da Bahia; 1995.

12. Cotrim HP, Paraná R, Portugal M, Santana N, Leal L, Cavalcante A, Lyra L. Esteato-hepatite não alcoólica: fatores predisponentes e principais características. GED. 1995;14(4):160.

13. Cotrim HP, Freitas LA, Paraná R, Portugal M, Lyra L, Andrade Z. Nonalcoholic steatohepatitis (NASH) and environmental toxins: a liver disease in workers from an industrial area. Hepatology. 1996;24(4):337A.

14. Cotrim HP, Paraná R, Portugal M, Lyra L, Andrade Z. Nonalcoholic steatohepatitis (NASH) and industrial toxins: follow up of patients removed from one industrial area. Hepatology. 1997;26(4):149A.

15. Cotrim HP, Andrade ZA, Paraná R, Portugal M, Lyra LG, Freitas LAR. Nonalcoholic steatohepatitis: a toxic liver disease in industrial workers. Liver. 1999;19(4):299-304.

16. Barberino JL, Carvalho FM, Silvany-Neto AM Gidi-de-Oliveira JF, Góes RC, Nascimento ALC, et al. Alterações hepáticas em traba- 
lhadores do refino de petróleo. Rev Baiana Saude Publica. No prelo.

17. Checkoway H, Pearce N, Kriebel D. Research methods in occupational epidemiology. $2^{\mathrm{a}}$ ed. New York: Oxford University Press; 2004.

18. Lwanga SK, Lemeshow S. Sample size determination in health studies. Genebra: WHO; 1991.

19. Masur J, Monteiro MG. Validation of the "CAGE" alcoholism screening test in a Brazilian psychiatric inpatient hospital setting. Rev Bras Med Pesq Biol. 1983;16(3):215-8.
20. Dean AG, Dean JA, Coulombier D, Brendel $\mathrm{KA}$, Smith DC, Burton AH, et al. Epi Info, Version 6.04a, a word processing, database, and statistics program for public health on IBMcompatible microcomputers. Atlanta: Centers for Disease Control and Prevention; 1996.

21. Hosmer Jr DW, Lemeshow S. Applied logistic regression. Nova Iorque: John Wiley \& Sons; 1989.

22. Oliveira NF, Santana VS, Lopes AAS. Razões de proporções e uso de método delta para in- tervalos de confiança em regressão logística. Rev Saude Publica. 1997;31(1):90-9.

23. SAS. Release 6.12. Cary: SAS Institute Inc.; 1996

24. SPSS for Windows. Release 6.1. Chicago: SPSS Inc.; 1994.

Manuscrito recebido em 31 de março de 2004. Aceito en versão revisada em 20 de julho de 2004.

ABSTRACT Objective. To determine the prevalence of liver changes in workers at an oil refinery located in the state of Bahia, Brazil, as compared to a reference population with no occupational exposure to chemical products, and to describe the factors associated with the observed differences in prevalence.

\section{Liver changes in workers at an oil refinery and in a reference population in the state of Bahia, Brazil}

Methods. We studied workers at the refinery and at the company's central management office located in the city of Salvador, which is the state capital. Blood samples of 692 refinery workers and 377 workers from the reference population were analyzed. Cases were defined as individuals presenting high serum levels of both gammaglutamyltransferase (GGT) and alanine aminotransferase (ALT) (GGT > 50 U/L for males and > $32 \mathrm{U} / \mathrm{L}$ for females; ALT > $50 \mathrm{U} / \mathrm{L}$ ). Data were collected on age, sex, weight, height, years working for the company, use of alcohol, smoking habits, physical exercise, occupational exposure to chemical products, use of personal safety equipment, and medical history of hepatitis, jaundice, and obesity.

Results. The prevalence of liver changes among refinery workers was $15.3 \%$ (95\% CI: 12.5 to 18.1 ), vs. $3.8 \%$ (95\% CI: 1,8 to 5,8) in the reference population. According to multiple logistic regression analysis, the prevalence of liver changes among refinery workers was 3.56 times greater $(95 \%$ CI: 1,99 to 6,38) than in the reference population, regardless of the presence of other relevant co-variables, such as obesity, exercise, smoking, and alcohol consumption.

Conclusions. These results suggest that occupational exposure may play a major role in the development of liver changes among workers at the oil refinery where the study was carried out.

Keywords Alanine aminotransferase, occupational exposure, gamma-glutamyltransferase. 